\title{
Aktuelle Studien zur Auswirkung der Psychomotorik-Therapie auf die kognitive Entwicklung
}

Iris Bräuninger

\section{Psychomotorische Aktivität und kognitive Entwicklung bei Kleinkindern}

$\mathrm{M}$ as und Riera (2017) überprüften in ihrer Interventionsstudie, wie die gewohnten Praktiken der Psychomotorik die kognitive Entwicklung von Kindern ab 12 Monaten steigern können. An der Studie nahmen 26 Kinder zwischen 12 und 22 Monaten teil, welche drei Gruppen (Go, G1 und $\mathrm{G}_{2}$ ) zugeteilt wurden. Die Studie bestand aus zwei Teilen: In der ersten 5-monatigen psychomotorischen Trainingsperiode erhielt $\mathrm{Go}(\mathrm{n}=11 \mathrm{Kin}-$ der) keine Psychomotorik-Sitzungen, $G_{1}(n=$ 8 Kinder) jede Woche eine Psychomotorik-Sitzung und $\mathrm{G}_{2}$ ( $\mathrm{n}=7$ Kinder) jede Woche zwei Psychomotorik-Sitzungen. Im zweiten Teil, der 23-monatigen Übungsperiode für Psychomotorik, erhielten alle drei Gruppen Go, G1 und G2 jede Woche eine Psychomotorik-Sitzung. Die 45-minütigen Psychomotorik-Sitzungen mit den drei Teilen (1) Begrüßung und Vorbereitung, (2) freies Spielen mit motorischen Bewegungen und (3) Abschluss, basierte auf der freien und spontanen Bewegung und der Interaktion der Kleinkinder mit ihrer Umgebung auf physischer, symbolischer und kognitiver Ebene (Aucouturiers 2004). Die Ergebnisse zeigten während der Trainingsperiode (Teil 1), dass die motorischen Fähigkeiten und kognitiven Bereiche in $G_{1}$ und $G_{2}$ höher als in $G_{0}$ waren. Während der Übungsperiode (Teil 2) zeigten sich Unterschiede zwischen den drei Gruppen im Bereich der Beweglichkeit, es gab jedoch keine Unterschiede im kognitiven Bereich. Die kognitiven Bereiche erreichten in den Gruppen $\mathrm{G}_{1}$ und $\mathrm{G}_{2}$ im Vergleich zu Go immer höhere Werte als die Bewegungsbereiche.

\section{Wirksamkeit von Psychomotorik auf} Körperschema und räumlich-zeitliche Struktur bei Kindern mit Downsyndrom

Die Wirksamkeit einer achtwöchigen Psychomotorik-Intervention (zu je ca. 40 min.) auf das Körperschema und die räumlich-zeitliche Struktur bei Kindern mit Downsyndrom wurde in der quasiexperimentellen Studie von Martínez und Antón (2018) mit Prä-Test-Post-Test-Design überprüft. An der Interventionsgruppe nahmen fünf Kinder teil (drei Jungen, zwei Mädchen, chronologisches Alter (CA) von 10,06-14,10 Jahren $(M=12,07$ und $S D=1,7)$, mentaler Altersbereich (MA) 45). Die Kontrollgruppe bestand ebenfalls aus fünf Kindern (drei Mädchen, zwei Jungen, CA-Bereich von 10; 00-14,06 Jahren $(M=11 ; 07$ und $S D=1 ; 8), M A<5)$. Im Prä-Test und Post-Test wurden die Kenntnisse des Körperschemas und die Anzahl der identifizierten Segmente des menschlichen Körpers sowie die Qualität und die motorische Unabhängigkeit ihrer Körperbewegungen in zwei nichtstandar- 
disierten Verfahren bewertet. Die Teilnehmerlnnen der PMT-Gruppe konnten ihr Wissen über ihren eigenen Körper und den anderer signifikant erweitern sowie ihr räumlich-zeitliches Wissen zur Körpererkennung ausbauen. Die motorische Aktivität schien sie ferner darin unterstützt zu haben, durch Bewegung die eigene Umgebung zu entdecken und leichter mit dieser zu kommunizieren. Dies werteten die Autorlnnen als grundlegend für die Verbesserung der psychologischen und motorischen Fähigkeiten sowie für den Erwerb neuen Lernens.

\section{Kognitive Fähigkeiten und frühe motorische Entwicklung von Vorschulkindern}

Osorio-Valencia, Torres-Sánchez, López-Carrillo, Rothenberg und Schnaas (2018) untersuchten die Bedeutung von Psychomotorik für die Entwicklung des Lernens in Bezug auf Lesen, Schreiben und mathematische Kalkulation. Hierzu analysierten sie Daten einer Kohortenstudie zur motorischen Leistung von dreijährigen Kindern, welche mit der Peabody Motor Scale bewertet wurden, sowie zu deren kognitiven Fähigkeiten im Alter von fünf Jahren. In die Kalkulation gingen Daten von 148 Kinder ein. Es wurden signifikante positive Assoziationen zwischen dem stationären Gleichgewicht und den verbalen Fähigkeiten im Alter von drei Jahren $(\beta=0,67, p=0,04)$ und dem Gedächtnis $(\beta=0,81, p=0,02)$ im Alter von fünf Jahren festgestellt: „Greifen und visuell-motorische Integration waren signifikant und positiv verbunden mit quantitativen Fähigkeiten $(\beta=0,74$, $p=0,005 ; \beta=0,61, p=0,01)$ und Gedächtnis ( $\beta=2,11, p=0,001 ; \beta=1,74, p=0,004$ )“ (Osorio-Valencia et al. 2018, 1015). Die Autorlnnen schlussfolgerten daraus, dass frühe motorische Leistung nach fünf Jahren zur Feststellung kognitiver Fähigkeiten und frühe motorische Stimulation zum Aufbau neuronaler Netzwerke beitragen können.

\section{Schlussfolgerung}

Alle Beiträge betonten die Wichtigkeit von Bewegung als Schlüssel für die physische und geistige Reifung. Bewegungsinterventionen scheinen beispielsweise eine wichtige Option für die Förderung der psychomotorischen Entwicklung bei Kindern mit Downsyndrom zu sein (Martínez / Antón 2018). Interessant ist bei diesen Beiträgen die Betonung der Rolle der Psychomotorik für die kognitive Entwicklung. Dies könnten wichtige Ergänzungen bei Diskussionen zur Schul-Curriculumsentwicklung und Revisionen werden. Weitere Studien scheinen für die Fragestellung „Kognitive Entwicklungsförderung durch Psychomotorik" sinnvoll und vielversprechend.

\section{Literatur}

Aucouturier, B. (2004): Los fantasmas de acción y la práctica psicomotriz (Vol. 7). Graó, Barcelona Martínez, V., Antón, A. (2018): Effects of a psychomotor intervention keywords: body schema down syndrome intervention program in preadolescents with down syndrome. Infancia y Aprendizaje 41 (1), 165-199

Mas, M., Riera, C. (2017): Systematization of the psychomotor activity and cognitive development. Educational Psychology 24 (1), 38-41

Osorio-Valencia, E., Torres-Sánchez, L., López-Carrillo, L., Rothenberg, S. J., Schnaas, L. (2018): Early motor development and cognitive abilities among Mexican preschoolers. Child Neuropsychology 24 (8), 1015-1025

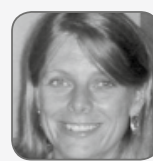

\section{Dr. Iris Bräuninger}

Senior Researcher \& Co-Leiterin Studiengang Psychomotoriktherapie (Hochschule für Heilpädagogik Zürich IVE), Dozentin MA Tanztherapie UAB Barcelona, BTD Supervisorin / Ausbilderin / Lehrtherapeutin, KMP Notatorin, Praxis Tanztherapie Supervision Bodensee.

\section{Dr. Iris Bräuninger} dancetherapy@mac.com und iris.braeuninger@hfh.ch 